\title{
Mineral balance, parathormone and vitamin D in patients with policystic ovary syndrome.
}

\author{
Adam Czyzyk ${ }^{1}$, Natalia Podkowa², Agnieszka Podfigurna-Stopa ${ }^{1}$, Blazej Meczekalski ${ }^{1}$
}

${ }^{1}$ Department of Gynecological Endocrinology, Poznan University of Medical Sciences, Poznan, Poland, 60-535

${ }^{2}$ Student Scientific Association of Gynecological Endocrinology, Poznan University of Medical Sciences

\section{Introduction}

Recent data showed that polycystic ovary syndrome (PCOS) is relate $d$ to abnormal calcium and phosphate metabolism. The patients are character zed by elevated levels of phosphorus and parathyroid hormone (PTH) ind decreased levels of vitamin $D$. Abnormalities in mineral homeostasis are relat $€ d$ to insulin resistance.

In this study we assessed a complex of calcium and phosphate metabolism indices in patients diagnosed with PCOS.

\section{Methods}

\section{Materials}

PCOS 52 women, aged $26 \pm 6$ years diagnosed with PCOS according to Rotterdam criteria were

Controls 24 healthy women aged $27 \pm 5$ years

\section{Calcium \&}

phosphorus concentrations were measured in:

- serum

- 24 hours urine collection

- fractional urine excretion was assessed.

We also measured the 250H-vitamin D3, PTH, gonadotropins, estradiol, testosterone, and lipid fraction concentrations.

Serum insulin and glucose concentrations were used to calculate the HOMA index.

\section{Results}

PCOS patients presented typical hormonal disturbances for PCOS with higher LH and testosterone serum levels and lower estradiol serum concentrations in comparison to controls.

Total serum calcium levels were higher in PCOS patients $(2.38 \pm 0.07 \mathrm{vs} 2.31 \pm 0.09 \mathrm{mmol} / \mathrm{L}, \mathrm{p}=0.007)$ but were within normal range.

Urine calcium and phosphorus concentrations were higher in PCOS group:

Calcium: $2.87 \pm 1.19$ in PCOS vs $2.42 \pm 0.96 \mathrm{mmol} / \mathrm{L}$ in controls, $\mathrm{p}=0.050$,

Phosphorus: $47.20 \pm 23.05$ in PCOS vs $37.11 \pm 12.65 \mathrm{mg} / \mathrm{dL}$ in controls, $\mathrm{p}=0.013$.

The other mineral concentrations and urine exertion were the same in both groups and within normal limits.

Urine calcium levels were positively correlated with HOMA and insulin concentrations (see graph 1. and 2.)

Serum Vitamin D and PTH concentrations were the same for both groups. Levels of vitamin D were below normal range in both groups $(16.8 \pm 9.3$ in PCOS vs $17.0 \pm 7.6$ in controls $p=0.53)$

PCos group

$$
\text { C }
$$

